\title{
A case presentation of an IgA nephropathy patient with Vogt-Koyanagi-Harada syndrome
}

\author{
Quan Zhang ${ }^{1,2} \mathbb{B}$, Xing Fan ${ }^{3}$, Meng $\operatorname{Tian}^{4}$ and Hongling $\operatorname{Han}^{1 *}$ (D)
}

\begin{abstract}
Background: Vogt-Koyanagi-Harada syndrome is a rare disease characterized by skin and eyelash bleaching, chronic granulomatous iridocyclitis and exudative retinal detachment, and aseptic meningitis and encephalopathy. IgA nephropathy complicated by Vogt-Koyanagi-Harada syndrome is very rare, even though they might have similar pathogeneses. Ocular lesions often are not examined when patients are diagnosed with IgA nephropathy, which affects the prognosis.

Case presentation: We describe a 55-year-old male IgA nephropathy patient who was admitted with high fever and hematuria. Physical examination revealed impaired binocular vision with blurred vision, impaired hearing, and a congestive rash on the chest and back. Renal ultrasound examination showed no abnormalities. Laboratory examination showed that glomerulonephritis was complicated by infection, and anti-infection therapy was ineffective. Bilateral fluorescein angiography showed Vogt-Koyanagi-Harada syndrome. Further renal biopsy confirmed IgA nephropathy. Hormone shock therapy and cyclophosphamide adjuvant therapy were administered, and the patient's symptoms improved.

Conclusion: For the first time, we reported the case of simultaneous onset of IgA nephropathy and Vogt-KoyanagiHarada syndrome, which is very rare. The onset of Vogt-Koyanagi-Harada syndrome is rapid and serious, while that of IgA nephropathy is relatively milder, making it easy for specialized doctors to neglect this condition. Doctors should be highly alert to the clinical concomitant occurrence of the two diseases with similar mechanisms, especially in the case of neurological defects and ocular symptoms in IgA nephropathy patients, since timely immunosuppressive treatment may improve the outcome of ocular diseases.
\end{abstract}

Keywords: IgA nephropathy, Vogt-Koyanagi-Harada syndrome, Human leukocyte antigen, Case presentation

\section{Background}

IgA nephropathy (IgAN) is common worldwide, especially in China. It is characterized by the varying extent of mesangial proliferation and mesangial immune complex deposition, with IgA as the predominant or codominant immunoglobulin type [1]. IgAN is restricted to the kidneys in most cases; however, it is sometimes

\footnotetext{
* Correspondence: hanhongling_zyy@163.com

'Department of Nephrology, Tianjin Medical University General Hospital, Tianjin, China

Full list of author information is available at the end of the article
}

accompanied by other conditions, such as ankylosing spondylitis, celiac disease, alcoholic and nonalcoholic liver disease, sarcoidosis, and dermatitis herpetiformis. The most common concomitant injury of the eyes in IgAN patients is scleritis, but ocular involvement is infrequent.

Vogt-Koyanagi-Harada syndrome (VKHS) is an inflammatory disease resulting in damage to multiple systems in the body, including granulomatous uveitis and meningeal stimulation, with or without auditory dysfunction, skin or hair changes [2]. VKHS and IgAN

(c) The Author(s). 2020 Open Access This article is licensed under a Creative Commons Attribution 4.0 International License, which permits use, sharing, adaptation, distribution and reproduction in any medium or format, as long as you give appropriate credit to the original author(s) and the source, provide a link to the Creative Commons licence, and indicate if changes were made. The images or other third party material in this article are included in the article's Creative Commons licence, unless indicated otherwise in a credit line to the material. If material is not included in the article's Creative Commons licence and your intended use is not permitted by statutory regulation or exceeds the permitted use, you will need to obtain permission directly from the copyright holder. To view a copy of this licence, visit http://creativecommons.org/licenses/by/4.0/. The Creative Commons Public Domain Dedication waiver (http://creativecommons.org/publicdomain/zero/1.0/) applies to the data made available in this article, unless otherwise stated in a credit line to the data. 
might have similar pathogeneses, but cases of VKHS combined with IgAN are rarely reported. We encountered one patient who developed VKHS with IgAN at the same time.

\section{Case presentation}

A 55-year-old man was admitted to our department with fever for 10 days and gross hematuria for 9 days. The patient had developed fever with a maximum temperature of $39^{\circ} \mathrm{C}$, without chills, abdominal pain or diarrhea 10 days prior. The temperature returned to normal after antipyretic and antibiotic administration under the guidance of community doctors, but it increased again and was accompanied by gross hematuria just a few hours later. The patient gradually developed foamy urine with eye pain, photophobia, tinnitus, rash and other discomforts before this recent admission to our department.

Physical examination revealed increased temperature $\left(37.7^{\circ} \mathrm{C}\right)$, normal blood pressure (Bp, $100 / 80 \mathrm{mmHg}$ ), and bradycardia (HR, $56 \mathrm{bpm})$. There was hyperemia on his head, neck and chest skin and hyperemia and rash on his back. His physical exam was otherwise unremarkable. Specifically, he had no oral lesions, lymphadenopathy, joint swelling, lower extremity edema, or cardiac murmur or rub.

A history of renal lithiasis for 3 years could be elicited. The diagnosis of renal lithiasis was established at the local hospital, and the patient had received extracorporeal lithotripsy at the same hospital. The patient denied eye trauma and a history of surgery. He was unaware of any familial genetic disease and had not been tested for a genetic disease.

The results of the initial laboratory tests were as follows: white blood cell count $15.0 \times 109 / \mathrm{L}$, neutrophil ratio $86.3 \%$, hemoglobin $118 \mathrm{~g} / \mathrm{L}$, platelets $215 \times 109 / \mathrm{L}$; albumin $24 \mathrm{~g} / \mathrm{L}$, blood urea nitrogen $6.4 \mu \mathrm{mol} / \mathrm{L}$, plasma creatinine $79 \mu \mathrm{mol} / \mathrm{L}$, uric acid $130 \mu \mathrm{mol} / \mathrm{L}$. blood sugar $5.8 \mu \mathrm{mol} / \mathrm{L}$. potassium $4.67 \mu \mathrm{mol} / \mathrm{L}$, sodium $142 \mu \mathrm{mol} / \mathrm{L}$, chloride $104 \mu \mathrm{mol} / \mathrm{L}$, calcium $2.11 \mu \mathrm{mol} / \mathrm{L}$, and $\mathrm{CO}_{2} \mathrm{CP}$ $25 \mu \mathrm{mol} / \mathrm{L}$. Urine erythrocytes $(6250.00 \mu \mathrm{L})$ and urinalysis showed 3+ blood and 1+ protein with 8-10 white blood cells/high-power field. Twenty-four-hour urine collection revealed $0.673 \mathrm{~g}$ of protein. Tests for the presence of rheumatoid factor, ANA and ANCA were negative. Tests for the presence of anti-mitochondrial and anti-smooth-muscle antibodies were negative. Complement levels were normal.

Symptoms gradually worsened after admission, and the body temperature fluctuated at $39-41{ }^{\circ} \mathrm{C}$ with more hematuria and more extensive rash. In addition, the patient developed a severe headache, neck stiffness, eye pain, conjunctival congestion, and decreased vision in both eyes, accompanied by tinnitus and hearing loss. The hearing test showed severe neurological deafness in both ears. The slit lamp examination showed that the conjunctiva of both eyes was mixed and congested, and mutton-fat-like KPs were observed, but the cornea was still clear. Bilateral intraocular pressure increased to 30 $\mathrm{mmHg}$ in the left and $35 \mathrm{mmHg}$ in the right. Fluorescein angiography revealed multiple leakages from the optic discs as well as the retinal pigment epithelium in the posterior pole of both eyes and the accumulation of cystic fluorescein, typical of Vogt-Koyanagi-Harada syndrome, as shown in Fig. 1.

A percutaneous renal biopsy was then performed. Sampling consisted of 17 glomeruli with 2 small cellular crescents with fibrinoid necrosis and 2 ischemic sclerosis and fuchsinophilic protein deposition in the mesangial area. Lymphocytes and monocytes showed multifocal and flaky infiltration in the renal interstitium with fibrosis. Immunofluorescent examination revealed strong IgA $(3+)$ deposition along the glomeruli, as well as $\operatorname{IgG}(+), \operatorname{IgM}(-), \mathrm{C} 3(++$ ), $\mathrm{C} 1 \mathrm{q}(-)$, and FRA(++). Based on the findings mentioned above, the diagnosis of IgA nephropathy (M1E0S1T1C1) with VKHS was confirmed, as shown in Fig. 2.

During hospitalization, the patient was given a bolus of methylprednisolone $500 \mathrm{mg} 3$ times, followed by treatment with prednisone $60 \mathrm{mg}$, mannitol and cyclophosphamide. The patient's symptoms were significantly relieved, intraocular pressure was reduced to normal, urinary protein was reduced to $208 \mathrm{mg} / 24 \mathrm{~h}$, and red blood cells were reduced to $1.90 \mu \mathrm{L}$ by contrast microscopy.

The patient is still taking prednisone and intermittent cyclophosphamide treatment, the urine test results have returned to normal and his condition is stable, without complaints of blurred vision or headache.

\section{Discussion and conclusion}

Vogt-Koyanagi-Harada syndrome is a rare multisystemic disease that affects tissues containing melanin, such as the eye, inner ear, meninges, and skin [3]. VKHS is predominantly found in patients aged between 20 and 50 years. The disease occurs more frequently among Asian, Native American, and Hispanic populations. VKHS is one of the most common causes of uveitis in Japan and Brazil [2]. Although the exact etiology of VKHS remains unclear, many studies have suggested that it is associated with genetics and the autoimmune system. VKHS is thought to be a Tcell-mediated autoimmune process directed against one or more antigenic components of melanocytes. CD4+ T cells, Th1 cytokines and HLA-DRB1*0405 play the main role in the pathogenesis of VKHS $[4,5]$. Previous reports have shown that $\mathrm{CD} 4+\mathrm{T}$ cells sensitive to melanocytes are responsible for the development of VKHS [6]. Viruses, such as the Epstein-Barr virus and cytomegalovirus, have been hypothesized as possible triggering factors of these mechanisms [7]. The disease is characterized by chronic bilateral panuveitis associated with a varying constellation 


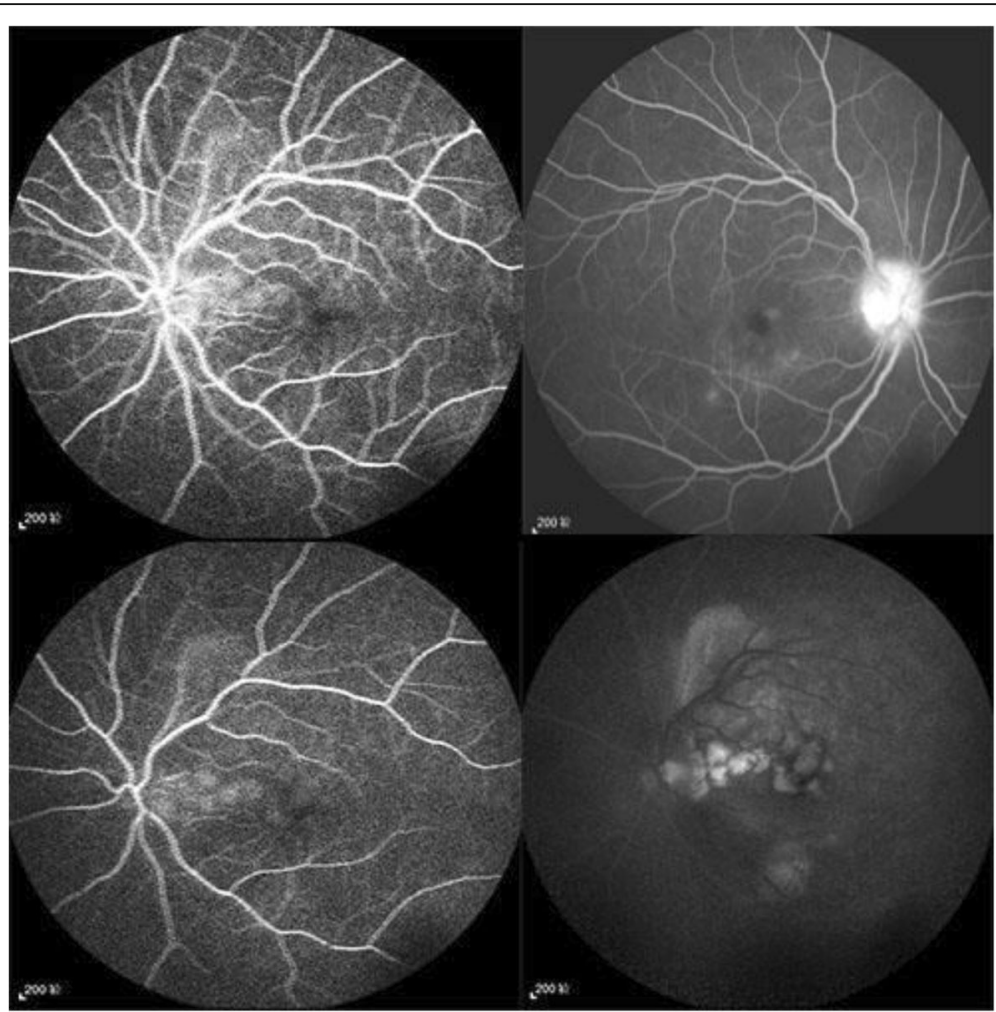

Fig. 1 Fluorescein angiography revealed multiple leakages from the optic discs as well as the retinal pigment epithelium in the posterior pole of both eyes and the accumulation of cystic fluorescein

of auditory, neurological and cutaneous manifestations [8]. The classic clinical course of VKHS can be divided into 4 stages: the prodromal stage, the acute uveitis stage, the convalescence stage, and the chronic recurrent stage in some patients [8].

Previous studies have found that VKHS can also be associated with other autoimmune disorders, such as ulcerative colitis, Crohn's disease, IgAN, autoimmune polyglandular syndrome type 1 , hypothyroidism, diabetes mellitus, and Hashimoto's thyroiditis. As far as we know, only 2 cases of IgA nephropathy complicated by this syndrome were reported in Japan in 2007 [9]. At the initial diagnosis of IgA nephropathy by renal biopsy, one patient showed low disease activity with low-grade mesangial cell proliferation, without hormone therapy, and VKHS appeared 11 years later. The other patient presented high pathological activity with glomerular crescent formation, which was treated with pulse corticosteroid therapy with oral prednisolone tapering, and presented the syndrome again 5 years later. IgA nephropathy was stable in both patients without corticosteroid treatment at the time of eye disease presentation.

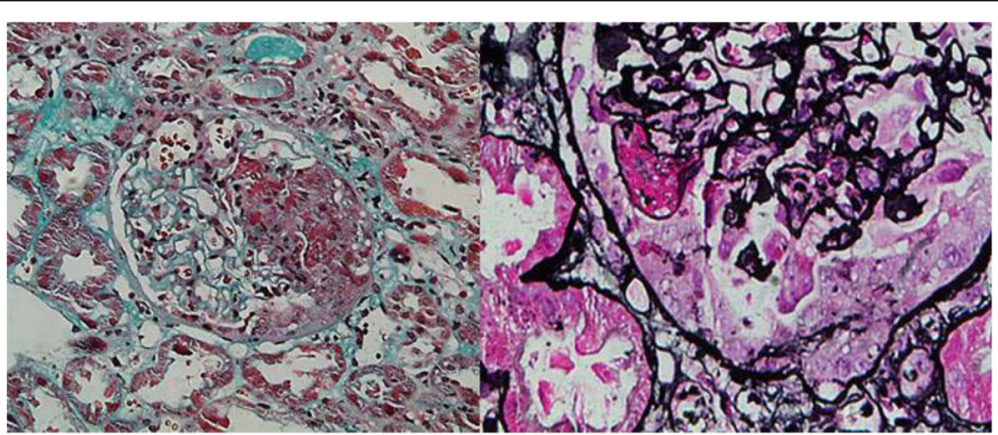

Fig. 2 Renal biopsy showed 17 glomeruli, among which 2 small cellular crescent bodies with fibrinoid necrosis were observed as well as renal interstitial fibrosis. Immunofluorescence indicated IgA+++, IgG+, IgM-, C3++, C1q-, and FRA++ deposits along the glomeruli, consistent with IgA nephropathy with focal hyperplasia and necrosis 
However, in our case, the patient had no previous extrarenal manifestations of IgAN or discomfort with eye diseases and presented hematuria, proteinuria and fever at the onset, accompanied by discomforts such as blurred vision and hearing loss. The renal manifestations and systemic lesions occurred at the same time, and the pathological manifestations were highly active. In consideration of the patient's condition, pulse corticosteroid therapy and steroid tapering were administered, with the addition of cyclophosphamide treatment. After treatment, the patient's vision and hearing were restored, and the symptoms of renal damage were reduced, which was consistent with previous literature reports [10].

Interestingly, our patient represents the first case diagnosed with both VKHS and severe IgAN, which may or may not be an incidental association. We believe that immunological mechanisms probably play an important role in this association.

Both Vogt-Koyanagi-Harada syndrome and IgA nephropathy are well known to be sometimes preceded by common cold-like symptoms. Virus infection (EB, CMV) may be involved in both diseases simultaneously as a trigger of further abnormal immune mechanisms [11]. VKHS is a systemic disease with an abnormal immune response of $\mathrm{T}$ cells. CD4+ $\mathrm{T}$ cells, $\mathrm{T}$ helper 17 (Th17) cells and regulatory $\mathrm{T}$ (Treg) cells play an important role in VKHS activities. The activation of C3aR in CD4+ T cells can upregulate the production of inflammatory factors such as IL-17, and the increased expression of C3aR may also lead to the overactivation of the Th17 cell response and promote the occurrence and development of VKHS [12]. However, the dysregulation of CD4+ T cell subsets also participates in the pathogenesis of IgAN. IgAN is characterized by higher proportions of circulatory Th2, Th17, and Th22 cells but lower proportions of Th1 and Treg cells. An imbalance of the ratio of Treg cells to Th17 cells is related to the pathogenesis of IgAN and is correlated with clinical severity. In IgAN, IL-4 (Th2 type IL), IL-17 (Th17 type IL) and TGF- $\beta$ may inhibit the expression of C1GALT1 and its chaperone (C1GALT1C1), which is mainly responsible for the addition of $\mathrm{N}$-acetylgalactosamine (GalNAc) to serine or threonine in the hinge region. The dysfunction of this process is an important mechanism for the formation of glycosylated abnormal IgA1 [13].

More incredibly, both diseases show immunological abnormalities and have genetic predispositions, such as familial occurrence and strong association with human leukocyte antigens (HLAs). IgAN is closely related to the HLA-DQB1, HLA-DRB1, HLA-DP, and DEFA alleles and shares most alleles with other immune system diseases, including Crohn's disease, ulcerative colitis, and SLE [14]. Coincidentally, VKHS is also closely related to these diseases, and HLA-DRB1*0405 is also the main susceptibility allele of VKHS [15]. These findings suggest that there may be an internal link between the two diseases that is still unclear and needs to be explored further.

For the first time, we reported the case of simultaneous onset of IgAN and VKHS, and our data supplement those of previous cases. The onset of VKHS is rapid and serious, while that of IgAN is relatively milder, making it easy for specialized doctors to neglect this condition. Doctors should be highly alert to the clinical concomitant occurrence of two diseases with similar mechanisms, especially in the case of neurological defects and ocular symptoms in IgAN patients, since timely immunosuppressive treatment may improve the outcome of ocular diseases.

\section{Abbreviations \\ VKHS: Vogt-Koyanagi-Harada syndrome; IgAN: IgA nephropathy; HLA: Human leukocyte antigens}

\section{Acknowledgments}

We would like to thank the patient for giving his consent to use the results obtained by the kidney biopsy for research and clinical research purposes. We thank the members of our departments for helpful discussions.

\section{Authors' contributions}

M.T. treated the patient. H.L.H. and X.F. performed the histopathological analysis of the kidney biopsy. Q.Z. wrote the manuscript. All authors read and approved the final version of the manuscript.

\section{Funding}

None

\section{Availability of data and materials}

All data generated or analyzed during this study are included in this published article.

Ethics approval and consent to participate Not applicable.

\section{Consent for publication}

The patient gave written informed consent to use the results obtained by the kidney biopsy for research and clinical research purposes before the kidney biopsy. Furthermore, the patient provided written informed consent for the publication of this report and the associated images.

\section{Competing interests}

The authors declare no conflicts of interest. The results presented in this paper have not been published previously in whole or part anywhere else.

\section{Author details}

'Department of Nephrology, Tianjin Medical University General Hospital, Tianjin, China. ${ }^{2}$ School of Graduate, Tianjin Medical University, Tianjin, China. ${ }^{3}$ Department of Nephrology, Tianjin 4th Central Hospital, Tianjin, China.

${ }^{4}$ Tianjin Jinnan Hospital, Tianjin, China.

Received: 30 October 2019 Accepted: 8 July 2020

Published online: 13 July 2020

\section{References}

1. Trimarchi H, Coppo R. Podocytopathy in the mesangial proliferative immunoglobulin a nephropathy: new insights into the mechanisms of damage and progression. Nephrol Dial Transplant. 2019;34(8).

2. Greco A, Fusconi M, Gallo A, Turchetta R, Marinelli C, Macri GF, et al. VogtKoyanagi-Harada syndrome. Autoimmun Rev. 2013;12:1033-8.

3. Silpa-Archa S, Silpa-Archa N, Preble JM, Foster CS. Vogt-Koyanagi-Harada syndrome: perspectives for immunogenetics, multimodal imaging, and therapeutic options. Autoimmun Rev. 2016;15:809-19. 
4. Abad S, Wieërs G, Colau D, Wildmann C, Delair E, Dhote R, et al. Absence of recognition of common melanocytic antigens by $T$ cells isolated from the cerebrospinal fluid of a Vogt-Koyanagi-Harada patient. Mol Vis. 2014;20:956-69.

5. Liang L, Peng XY, Wang $H$. Th lymphocyte subsets in patients with VogtKoyanagi-Harada disease. Int J Ophthalmol. 2019;12:207-11.

6. Fang W, Yang P. Vogt-koyanagi-harada syndrome. Curr Eye Res. 2008;33: 517-23.

7. Bassili SS, Peyman GA, Gebhardt BM, Daun M, Ganiban GJ, Rifai A. Detection of Epstein-Barr virus DNA by polymerase chain reaction in the vitreous from a patient with Vogt-Koyanagi-Harada syndrome. Retina. 1996;16:160-1.

8. Sakata VM, da Silva FT, Hirata CE, de Carvalho JF, Yamamoto JH. Diagnosis and classification of Vogt-Koyanagi-Harada disease. Autoimmun Rev. 2014; 13:550-5.

9. Matsuo T, Masuda I, Ota K, Yamadori I, Sunami R, Nose S. Vogt-KoyanagiHarada syndrome in two patients with immunoglobulin a nephropathy. Acta Med Okayama. 2007:61:305-9.

10. Du L, Kijlstra A, Yang P. Vogt-Koyanagi-Harada disease: novel insights into pathophysiology, diagnosis and treatment. Prog Retin Eye Res. 2016;52:84-111.

11. Ito R, Ota M, Meguro A, Katsuyama Y, Uemoto R, Nomura E, et al. Investigation of association between TLR9 gene polymorphisms and VKH in Japanese patients. Ocul Immunol Inflamm. 2011;19:202-5.

12. Wang C, Cao S, Zhang D, Li H, Kijlstra A, Yang P. Increased complement 3a receptor is associated with Behcet's disease and Vogt-Koyanagi-Harada disease. Sci Rep. 2017;7:15579.

13. Ruszkowski J, Lisowska KA, Pindel M, Heleniak Z, Dębska-Ślizień A, Witkowski JM. T cells in IgA nephropathy: role in pathogenesis, clinical significance and potential therapeutic target. Clin Exp Nephrol. 2019;23:291-303.

14. Shi $M$, Ouyang $Y$, Yang $M$, Yang $M$, Zhang $X$, Huang $W$, et al. IgA nephropathy susceptibility loci and disease progression. Clin J Am Soc Nephrol. 2018;13:1330-8.

15. Shi T, Lv W, Zhang L, Chen J, Chen H. Association of HLA-DR4/HLA-DRB1*04 with Vogt-Koyanagi-Harada disease: a systematic review and meta-analysis. Sci Rep. 2014;4:6887.

\section{Publisher's Note}

Springer Nature remains neutral with regard to jurisdictional claims in published maps and institutional affiliations.

Ready to submit your research? Choose BMC and benefit from:

- fast, convenient online submission

- thorough peer review by experienced researchers in your field

- rapid publication on acceptance

- support for research data, including large and complex data types

- gold Open Access which fosters wider collaboration and increased citations

- maximum visibility for your research: over $100 \mathrm{M}$ website views per year

At $\mathrm{BMC}$, research is always in progress.

Learn more biomedcentral.com/submissions 